\title{
Correction to: On the unitary block-decomposability of 1-parameter matrix flows and static matrices
}

\section{Frank Uhlig ${ }^{1}$}

Published online: 27 October 2021

(C) Springer Science+Business Media, LLC, part of Springer Nature 2021

\section{Correction to: Numerical Algorithms https://doi.org/10.1007/s11075-021-01124-7}

This paper suffers from a typo like error that uses the adjective 'diagonalizable' several times on two adjacent pages, thereby falsifying the results badly. This is mitigated as follows :

(A) On p. 5, line 18; the first line of Theorem 2 should read:

'A general complex time-varying matrix flow $A(t)$ can be properly and uniformly block-diagonalized by ...',

with the second printed word 'diagonalizable' removed because it is erroneous and falsifies the result.

(B) On p. 6, line 1; the first line of Theorem 3 should read:

'A general complex matrix $A$ can be properly block-diagonalized by ...', with the second printed word 'diagonalizable' removed because it is in error and falsifies the result.

(C) On p 5, lines 13 - 15; in the paragraph following Theorem 1, the first sentence should read:

'For general complex matrix flows $A(t)_{n, n}$ the same invariant subspace argument holds, except that the unitary eigenvector matrix $\tilde{V}\left(t_{a}\right)$ similarity needs to be replaced by ...',

with the sub-clause 'that are diagonalizable throughout their single-parameter range,' removed since it is confusing and restricts the global result needlessly.

The original article can be found online at https://doi.org/10.1007/s11075-021-01124-7.

Frank Uhlig

uhligfd@auburn.edu

1 Department of Mathematics and Statistics, Auburn University, Auburn, AL 36849, USA 
(D) On p. 6, lines $-6,-5$; the sentence near the bottom of the page just above the displayed item (I) should read :

'As theory tells us, to solve the general matrix flow decomposability problem it suffices to compute ...',

followed on a new line as before by : '(I) the eigenvector matrix ...'

This simplifies the sentence and removes the nonsensical 'diagonalizable' mention.

These four errors occurred unknowingly in the final proofing and the author apologizes for these unintended mishaps. All results and proofs of the paper pertain to general matrix flows and general static matrices. None of these need to be 'diagonalizable' which is totally irrelevant here.

Publisher's note Springer Nature remains neutral with regard to jurisdictional claims in published maps and institutional affiliations. 